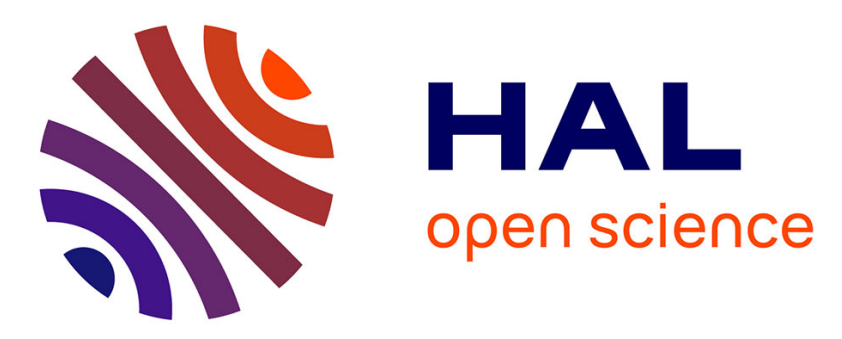

\title{
Status of Virgo detector
}

F. Acernese, P. Amico, M. Alshourbagy, F. Antonucci, S. Aoudia, P. Astone, S. Avino, D. Babusci, G. Ballardin, F. Barone, et al.

\section{To cite this version:}

F. Acernese, P. Amico, M. Alshourbagy, F. Antonucci, S. Aoudia, et al.. Status of Virgo detector. 11th Gravitational Waved Data Analysis Workshop, 2006, Potsdam, Germany. pp.S381-S388, 10.1088/0264-9381/24/19/S01 . in2p3-00175815

\section{HAL Id: in2p3-00175815 https://hal.in2p3.fr/in2p3-00175815}

Submitted on 8 Oct 2007

HAL is a multi-disciplinary open access archive for the deposit and dissemination of scientific research documents, whether they are published or not. The documents may come from teaching and research institutions in France or abroad, or from public or private research centers.
L'archive ouverte pluridisciplinaire HAL, est destinée au dépôt et à la diffusion de documents scientifiques de niveau recherche, publiés ou non, émanant des établissements d'enseignement et de recherche français ou étrangers, des laboratoires publics ou privés. 


\section{Status of Virgo detector}

F. Acernese $^{6}$, P. Amico ${ }^{10}$, M. Alshourbagy ${ }^{11}$, F. Antonucci ${ }^{12}$, S. Aoudia ${ }^{7}$, P. Astone ${ }^{12}$, S. Avino ${ }^{6}$, D. Babusci ${ }^{4}$, G. Ballardin ${ }^{2}$, F. Barone ${ }^{6}$, L. Barsotti ${ }^{11}$, M. Barsuglia ${ }^{8}$, F. Beauville ${ }^{1}$, S. Bigotta ${ }^{11}$, S. Birindelli ${ }^{11}$, M.A. Bizouard ${ }^{8}$, C. Boccara ${ }^{9}$, F. Bondu ${ }^{7}$, L. Bosi ${ }^{10}$, C. Bradaschia ${ }^{11}$, S. Braccini ${ }^{11}, A . B$ Brillet $^{7}$, V. Brisson ${ }^{8}$, D. Buskulic ${ }^{1}$, E. Calloni ${ }^{6}$, E. Campagna ${ }^{3}$,

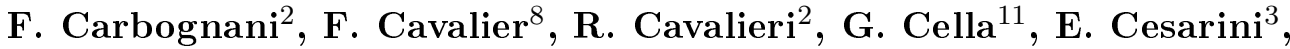
E. Chassande-Mottin ${ }^{7}$, N. Christensen ${ }^{2}$, C. Corda ${ }^{11}$, A. Corsi ${ }^{12}$, F. Cottone $^{10}$, A.-C. Clapson ${ }^{8}$, F. Cleva ${ }^{7}$, J.-P. Coulon ${ }^{7}$, E. Cuoco ${ }^{2}$,

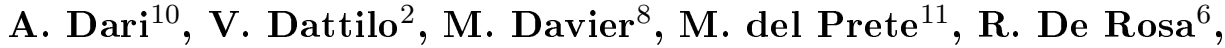
L. Di Fiore ${ }^{6}$, A. Di Virgilio ${ }^{11}$, B. Dujardin ${ }^{7}$, A. Eleuteri ${ }^{6}$, I. Ferrante ${ }^{11}$, F. Fidecaro ${ }^{11}$, I. Fiori ${ }^{11}$, R. Flaminio ${ }^{1,2}$, J.-D. Fournier ${ }^{7}$, S. Frasca ${ }^{12}$, F. Frasconi ${ }^{11}$, L. Gammaitoni ${ }^{10}$,

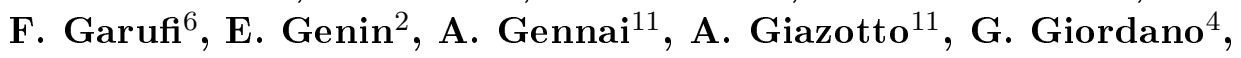
L. Giordano ${ }^{6}$, R. Gouaty ${ }^{1}$, D. Grosjean ${ }^{1}$, G. Guidi ${ }^{3}$, S. Hebri ${ }^{2}$, H. Heitmann ${ }^{7}$, P. Hello ${ }^{8}$, S. Karkar ${ }^{1}$, S. Kreckelbergh ${ }^{8}$,

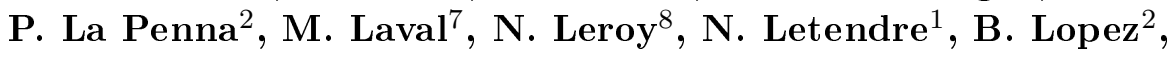
Lorenzini $^{3}$, V. Loriette ${ }^{9}$, G. Losurdo ${ }^{3}$, J.-M. Mackowski ${ }^{5}$, E. Majorana ${ }^{12}$, C. N. $\operatorname{Man}^{7}$, M. Mantovani ${ }^{11}$, F. Marchesoni ${ }^{10}$, F. Marion ${ }^{1}$, J. Marque ${ }^{2}$, F. Martelli ${ }^{3}$, A. Masserot $^{1}$, M. Mazzoni ${ }^{3}$, L. Milano ${ }^{6}$, F. Menzinger ${ }^{2}$, C. Moins ${ }^{2}$, J. Moreau ${ }^{9}$, N. Morgado ${ }^{5}$, B. Mours ${ }^{1}$, F. Nocera ${ }^{2}$, C. Palomba ${ }^{12}$, F. Paoletti ${ }^{2}, 11$, S. Pardi ${ }^{6}$, A. Pasqualetti ${ }^{2}$, R. Passaquieti ${ }^{11}$, D. Passuello ${ }^{11}$, F. Piergiovanni ${ }^{3}$, L. Pinard ${ }^{5}$, R. Poggiani ${ }^{11}$, M. Punturo ${ }^{10}$, P. Puppo $^{12}$, K. Qipiani ${ }^{6}$, P. Rapagnani ${ }^{12}$, V. Reita ${ }^{9}$, A. Remillieux ${ }^{5}$, F. Ricci ${ }^{12}$, I. Ricciardi ${ }^{6}$, P. Ruggi ${ }^{2}$, G. Russo ${ }^{6}$, S. Solimeno ${ }^{6}$, A. Spallicci ${ }^{7}$, M. Tarallo ${ }^{11}$, M. Tonelli ${ }^{11}$, A. Toncelli ${ }^{11}$, E. Tournefier ${ }^{1}$, F. Travasso ${ }^{10}$, C. Tremola $^{11}$, G. Vajente ${ }^{11}$, D. Verkindt ${ }^{1}$, F. Vetrano ${ }^{3}$, A. Viceré ${ }^{3}$, J.-Y. Vinet ${ }^{7}$, H. Vocca ${ }^{10}$ and M. Yvert $^{1}$

${ }^{1}$ Laboratoire d'Annecy-le-Vieux de Physique des Particules (LAPP), IN2P3/CNRS, Université de Savoie, Annecy-le-Vieux, France;

${ }^{2}$ European Gravitational Observatory (EGO), Cascina (Pi), Italia;

${ }^{3}$ INFN, Sezione di Firenze/Urbino, Sesto Fiorentino, and/or Università di Firenze, and/or Università di Urbino, Italia;

${ }^{4}$ INFN, Laboratori Nazionali di Frascati, Frascati (Rm), Italia;

${ }^{5}$ LMA, Villeurbanne, Lyon, France;

${ }^{6}$ INFN, sezione di Napoli and/or Università di Napoli "Federico II" Complesso Universitario di Monte S.Angelo, and/or Università di Salerno, Fisciano (Sa), Italia;

${ }^{7}$ Departement Artemis - Observatoire de la Côte d'Azur, BP 4220906304 Nice, Cedex 4, France;

${ }^{8}$ LAL, Univ Paris-Sud, IN2P3/CNRS, Orsay, France

${ }^{9}$ ESPCI, Paris, France; 
${ }^{10}$ INFN, Sezione di Perugia and/or Università di Perugia, Perugia, Italia;

${ }^{11}$ INFN, Sezione di Pisa and/or Università di Pisa, Pisa, Italia;

${ }^{12}$ INFN, Sezione di Roma and/or Università "La Sapienza", Roma, Italia.

E-mail: gabriele.vajente@sns.it

Abstract. The commissioning of the Virgo gravitational wave detector has restarted after several major hardware upgrades carried out during winter 2005. Now Virgo is fully operative and its sensitivity greatly improved and improving. A program of short scientific data taking has already started and Virgo is moving toward a period of continuous data taking, which should start at the end of May. The actual status of the Virgo detector is reported here, describing the actual detector sensitivity as well as the limiting noises and the mid-term plans.

PACS numbers: $04.80 . \mathrm{Nn}, 95.55 . \mathrm{Ym}$

\section{Introduction}

The Virgo gravitational wave detector, jointly funded by INFN (Italy) and CNRS (France) and located at the European Gravitational Observatory (EGO) near Pisa, is a power recycled Michelson interferometer with arms composed of $3 \mathrm{~km}$ long FabryPerot resonant cavities [1]. A schematic optical layout is shown in figure 1. The input beam is generated by a $20 \mathrm{~W}$ high-power $\mathrm{Nd}: \mathrm{YVO}_{4}$ laser locked to a $1 \mathrm{~W}$ solid state Nd:YAG master laser. The beam enters the vacuum and passes through a $144 \mathrm{~m}$ long input mode cleaner (IMC) which is used both for spatial filtering of the beam and for pre-stabilization of the laser frequency. A small fraction of the beam is sent to the rigid reference cavity (RFC), used as a frequency reference. The beam enters then the main interferometer, composed by a power recycling mirror (PR), a beam splitter (BS) and two resonant cavities with flat input mirrors (NI and WI) and curved terminal mirrors (NE and WE). The beams transmitted by the cavity end mirrors are sensed by both photodiodes and quadrant split photodiodes: the signals obtained from the former are used in the earlier stages of lock acquisition [3], while those from the latter are used for automatic alignment purposes, since in Virgo the main modulation frequency is chosen in order to be resonant in the recycling cavity and to make the upper sideband first transverse mode resonant in the arm cavities [4]. The beams reflected by the secondary anti-reflection coated surface of the beam splitter and by the power recycling mirror are also sent outside the vacuum and sensed by photodiodes and quadrant split photodiodes.

The main output beam coming from the recombination at the beam splitter passes through an output mode cleaner (OMC) which is suspended in vacuum, in order to improve the interferometer contrast by filtering out high order modes. The demodulation of this beam gives the gravitational wave output signal.

All mirrors are suspended to multi-stage passive seismic isolation system (superattenuators [2]) which can be controlled both by using local signals or global signals only, when the interferometer is in the final fully controlled condition. 


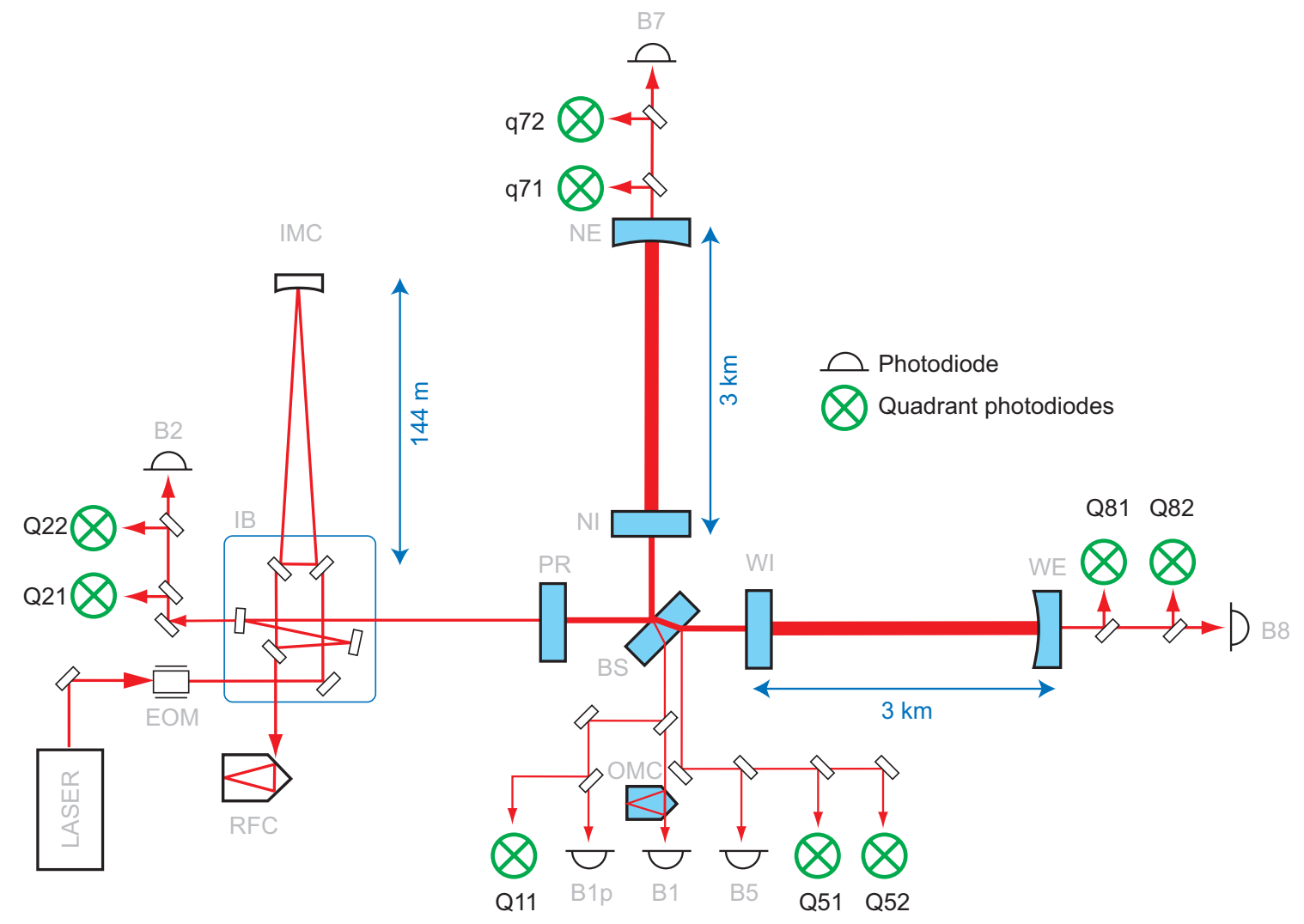

Figure 1. A simplified scheme of the Virgo optical layout. See the text for details.

\section{Hardware modification during winter 2005 shutdown}

A three months shutdown took place just after the $\mathrm{C} 7$ commissioning run, starting from mid September 2005 and ending in December of the same year. During this period two main hardware changes were carried out: the power recycling mirror and the whole suspended injection bench have been substituted.

\subsection{Power recycling mirror}

The power recycling mirror originally suspended was a compound one, with a curved secondary surface to form the last part of the beam matching telescope. The main drawback of this compound mirror was that it showed internal resonances between $100 \mathrm{~Hz}$ and $500 \mathrm{~Hz}$ that made the design of the ITF control system more difficult.

The new mirror is instead monolithic, flat and has a higher reflectivity $(95 \%$ instead of $92 \%$ ). Apart from the increased recycling gain expected due to the increased reflectivity, the new mirror allowed to strongly improve the performances of the longitudinal control system, due to the absence of internal resonances (see figure 2). 


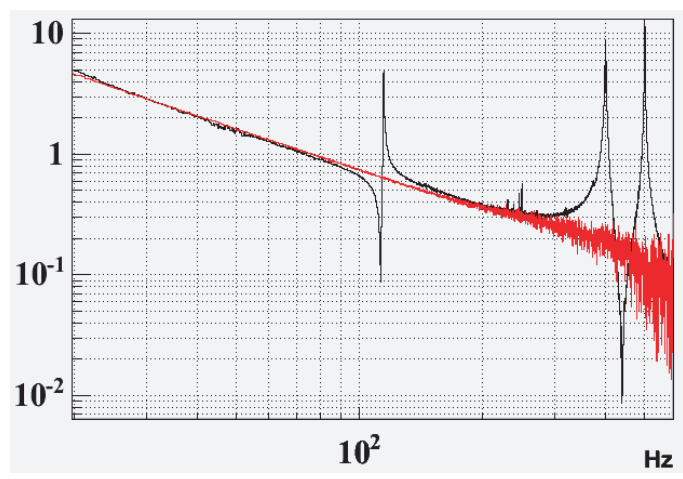

Figure 2. Comparison of the open loop transfer function for the longitudinal control of a cavity composed by the PR mirror and the NI mirror. The black curve shows a measurement performed with the old power recycling mirror, while the red curve is a measurement with the new mirror. Internal resonances of the compound PR mirror are well visible in the latter measurement.

\subsection{New suspended injection bench}

Before the shutdown, the suspended injection bench included a partially reflecting mirror which was used to attenuate the power entering the interferometer by a factor 10 . This was necessary to avoid back-reflected light to interfere inside the IMC, spoiling the control of this cavity and the level of laser frequency noise. The new injection bench has been designed including a Faraday insulator placed in transmission of the IMC, in order to inject the full power inside the ITF and to avoid the quoted problem. This insulator gives an attenuation of the beam coming back by a factor roughly 1000, which proved to be sufficient. Another feature of the new injection bench is the telescope used for matching the beam with the resonant mode of the interferometer: this telescope is composed of two parabolic mirrors and has been designed in order to reduce as much as possible the astigmatism of the beam. The last important point to note is the photodiode used for stabilizing the laser power, which is now placed on this suspended bench, in vacuum, in transmission of the IMC.

\subsection{Acoustic insulation}

During another shorter shutdown, in September 2006, an acoustic insulation shielding has been installed around the laser bench and the external injection bench. Several tests performed after this operation shows a relevant reduction of acoustic noise contamination of the dark fringe signal.

\section{Commissioning restart}

The main challenge in recovering a stable lock of the interferometer after these major changes was the appearance of thermal effects in the final steps of the lock acquisition sequence. When the interferometer is finally bring to dark fringe, meaning that the full 


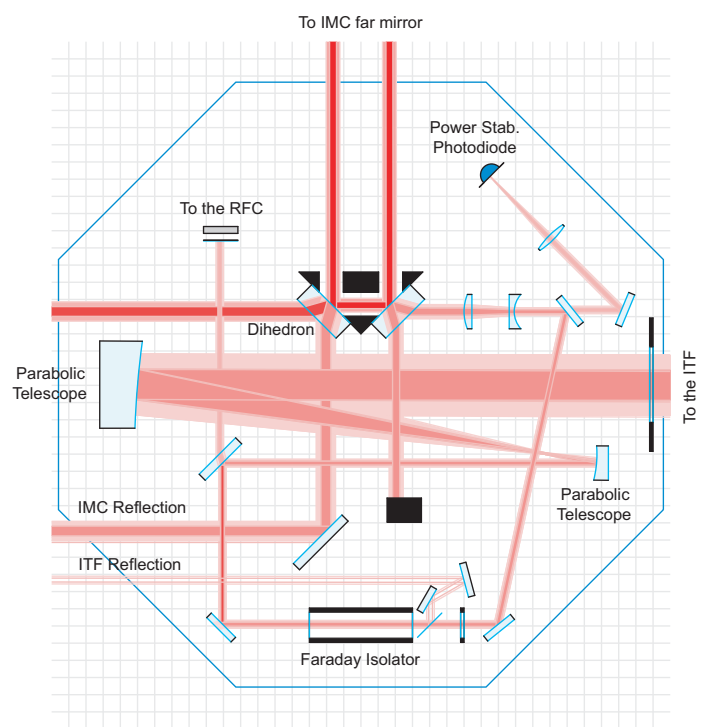

Figure 3. Simplified scheme of the new suspended injection bench. Note the mode matching parabolic telescope, the suspended photodiode for laser power stabilization placed in transmission of the IMC and the Faraday isolator.

power build-up takes place in the arm cavities, the two input mirrors start to heat up, changing the interferometer state and response. This effect made the development of a working lock acquisition strategy more difficult and the interferometer strongly sensitive to the amount of input power. We were forced to decrease the power injected inside the interferometer, which is now of the order of $5.3 \mathrm{~W}$. In this condition it is possible to acquire the lock, but the full sequence takes about 20 minutes, due to the need of waiting for the mirrors to thermalize.

\section{Week-end Science Runs}

From September 2006 a program of short science data taking (week-end science run, WSR) has started. In mean twice per month a full week-end, starting from Friday night until Monday morning, is dedicated to continuous data taking, with the interferometer locked in the best possible configuration. The main goals of this WSR program is to take periodic pictures of the state and sensitivity of the detector; to track the improvement in sensitivity, robustness and duty cycle; to collect some data in clean and controlled conditions, to be used for both commissioning and data analysis purposes; to start moving toward the organization needed for a longer science run. It must be noted that the priority remains always on the commissioning activities, and therefore a WSR run can be canceled or postponed if the detector is not in good working conditions or if there are other needs.

So far, ten week-end science run has been performed (refer to table 1 for their dates). Two of these has been canceled due to bad working condition of the interferometer. All the other runs had quite a good duty cycle and the longest lock obtained so far was 
Table 1. The following table lists the dates and duty cycles of past WSR runs.

\begin{tabular}{lll}
\hline Run & Date & Duty cycle \\
\hline WSR1 & September 08-11, 2006 & $88 \%$ \\
WSR2 & September 22-25, 2006 & $69 \%$ \\
WSR3 & October 06-09, 2006 & canceled \\
WSR4 & October 13-16, 2006 & canceled \\
WSR5 & November 10-13, 2006 & $62 \%$ \\
WSR6 & December 01-04, 2006 & $81 \%$ \\
WSR7 & January 12-17, 2007 & $76 \%$ \\
WSR8 & February 09-12, 2007 & $92 \%$ \\
WSR9 & February 17-19, 2007 & $100 \%$ \\
WSR10 & March 09-12, 2007 & $60 \%$ \\
\hline
\end{tabular}

that of WSR9, when the detector was stably locked for more than 55 hours. The main factor limiting the stability of the interferometer is linked to environmental conditions, mainly micro-seismic activity due to sea and wind. A lot of efforts has been devoted to improve this situation: during the first runs it was difficult to acquire the lock with strong wind and sea activity, while now the situation is much improved: the sensitivity is however still slightly varying in dependence of the micro-seismic conditions.

In figure 4 it is possible to see the improvements in sensitivity obtained during the last months, compared to that of the last Virgo commissioning run $\mathrm{C} 7$ which took place in September 2005. The improvements in the high frequency region (above $1 \mathrm{kHz}$ ) are due to the increased laser power when passing from C7 to WSR1 and to better matching of the beam and reduced frequency noise coupling for the other WSR runs. The improvements in an intermediate frequency region (between $200 \mathrm{~Hz}$ and $1 \mathrm{kHz}$ ) are due to the large efforts being carried out to reduce contamination by diffused and back-scattered light than can couple with acoustic noise and seismic vibrations of the external benches. The improvement between $50 \mathrm{~Hz}$ and $200 \mathrm{~Hz}$ between the first WSR runs and WSR7 is due to the use of a different and less noisy error signal for the control of one of the longitudinal degrees of freedom, namely the length of the power recycling cavity. Great improvements in the stability and accuracy of the angular control servos helped to reduce the low frequency RMS motion of the interferometer, allowing a better tuning of the longitudinal control systems. This resulted in the improvements visible between WSR7 and WSR8, when a frequency dependent noise subtraction technique has been used. Finally before the start of WSR10 run a source of electromagnetic noise has been found and reduced, resulting in the strong suppression of noise in the region around $100 \mathrm{~Hz}$.

\section{Noise performances}

There are several sources of noises that actually limit the sensitivity (see figure 5). At low frequency (between 10 and $50 \mathrm{~Hz}$ ) the dominating source of noise comes from the 


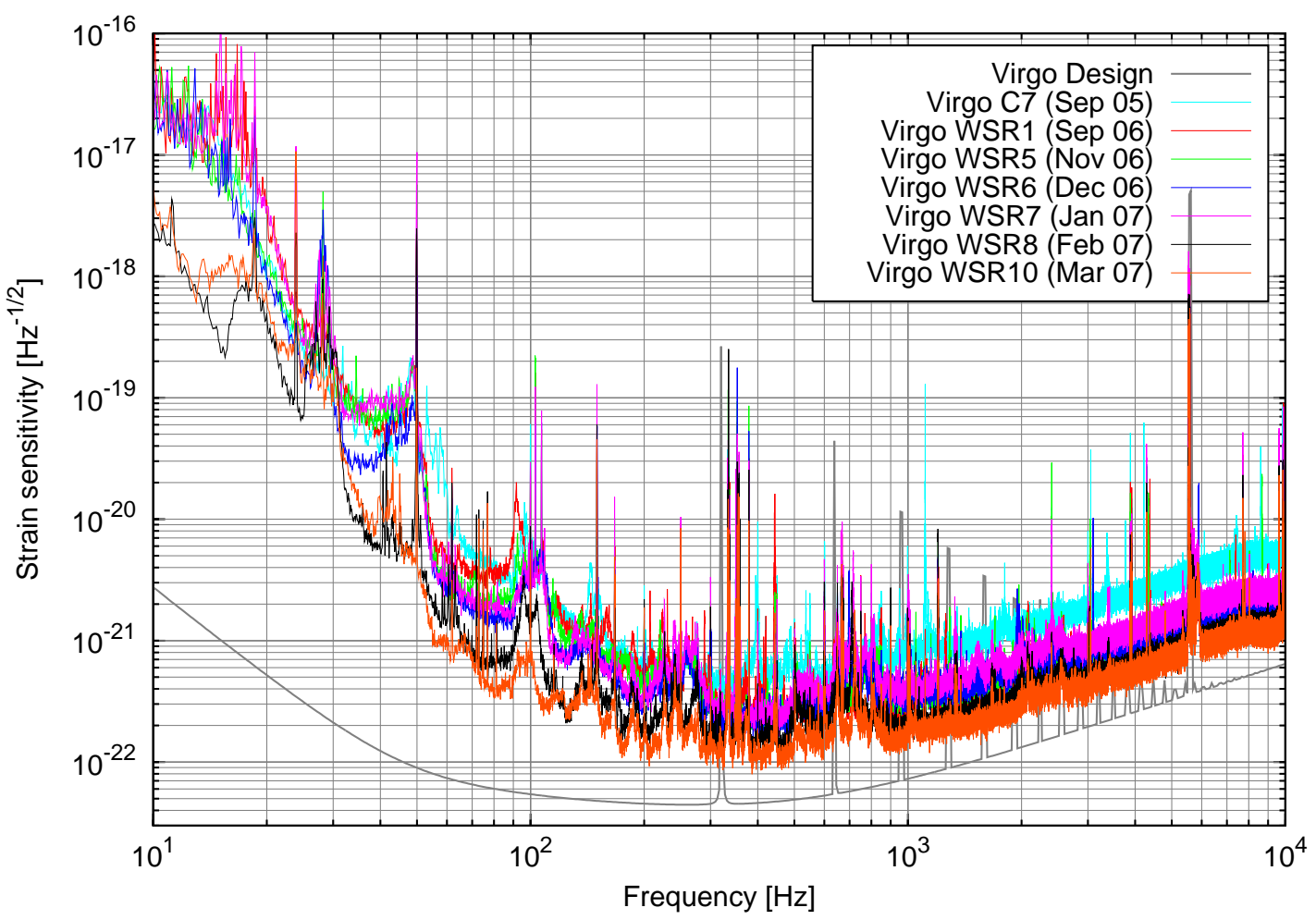

Figure 4. Strain sensitivity of the Virgo detector during some of the WSR runs, compared to that of the $\mathrm{C} 7$ commissioning run. The light gray curve is the Virgo design sensitivity.

longitudinal control servos, mainly the one controlling the short Michelson degree of freedom. Angular control noise is for the moment below the actual sensitivity, and for most of the angular degrees of freedom actually the contribution to dark fringe noise is even below the design sensitivity above 30-40 Hz. The noise at high frequency (above $1 \mathrm{kHz}$ ) is also quite well understood, and it comes mainly from shot noise at dark fringe and frequency noise in a small region around $6 \mathrm{kHz}$. The noise in the intermediate region, between $50 \mathrm{~Hz}$ an up to $1 \mathrm{kHz}$ is likely to be due to acoustic noise and seismic noise at the level of the external benches, reintroduced due to diffused and scattered light.

The stability and stationarity of the detector noise has also greatly improved during the last months, allowing stable and repeatable operation of the detector.

\section{Conclusions}

The Virgo detector sensitivity has reached a level compared to that of the LSC detectors above $500 \mathrm{~Hz}$ (see figure 6), and very close in the remaining part of the observational bandwidth. Therefore the main goal of the Virgo collaboration is to join the LSC detectors for a coincident data taking period. This first Virgo long science run is planned to start at the end of May and to continue for several months, at least until the end of 


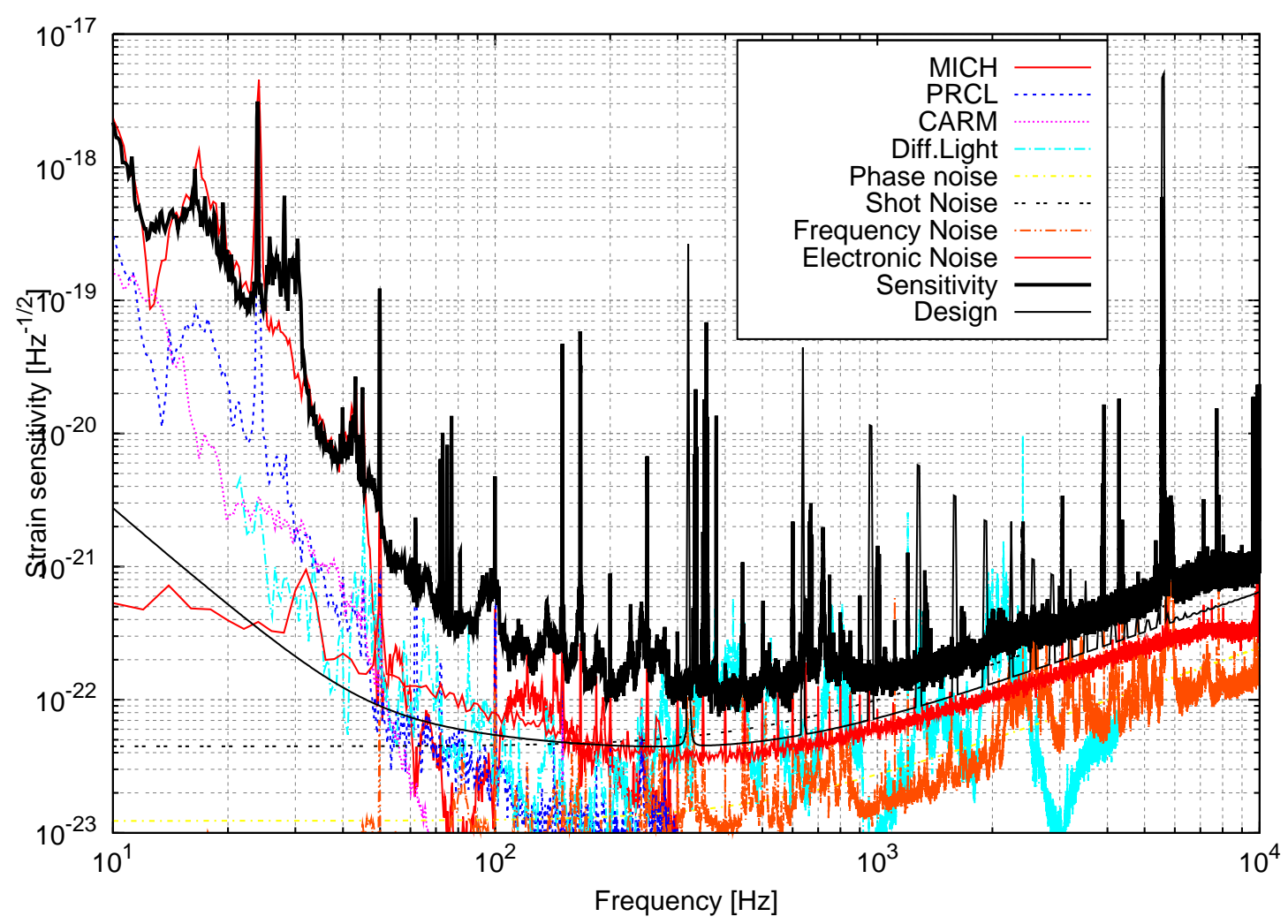

Figure 5. Noise budget for the WSR10 run. MICH, PRCL and CARM curves refers to longitudinal control noise, respectively for the short Michelson, power recycling cavity length and long cavities common mode degrees of freedom. The shot noise is computed taking into account the actual power reaching the dark fringe port. Electronic and phase noise are estimated using analytical models. The diffused light curve shows an example of projections of acoustic and seismic excitation of external benches, estimated using noise injection.

the LIGO S5 run.

Before the beginning of the science run, the main task for the commissioning remains to improve as much as possible the detector sensitivity and duty cycle. Main activities concerns control noise characterization and possibly reduction for the low frequency region; acoustic shielding installation in the detection laboratory and substitution of sensitive optical element to reduce acoustic and seismic noise contamination due to diffused and scattered light; implementation of a new electronic for the laser frequency stabilization servo.

These modifications should allow to further improve the sensitivity, reaching the level of the other detectors even in the frequency region between $50 \mathrm{~Hz}$ and $500 \mathrm{~Hz}$.

[1] http://www.virgo.infn.it

[2] F. Acernese et al. [Virgo Collaboration], "Measurement of the seismic attenuation performance of the Virgo Superattenuator", Astroparticle Phys. 23 (2005) 557-565

[3] F. Acernese et al. [Virgo Collaboration], "The variable finesse locking technique", Class. Quantum Grav. 23 (2006) S85. 


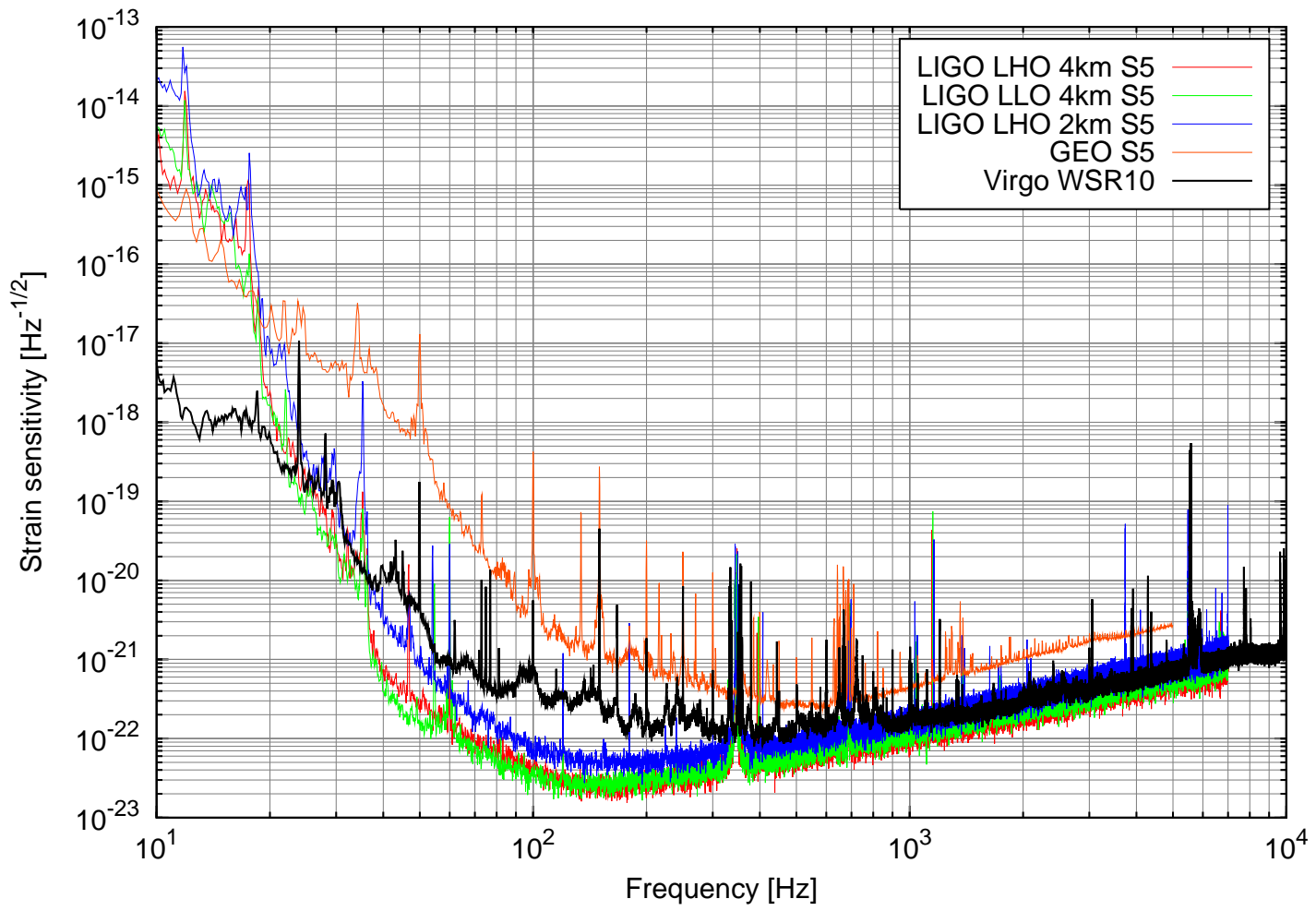

Figure 6. Comparison of the Virgo sensitivity during WSR10 with that of the LSC detectors during S5 run.

[4] F. Acernese et al. [Virgo Collaboration], "The Virgo automatic alignment system", Class. Quantum Grav. 23 (2006) S91. 